PROCEEDINGS OF THE

AMERICAN MATHEMATICAL SOCIETY

Volume 127, Number 6, Pages 1641-1650

S 0002-9939(99)04811-X

Article electronically published on February 18, 1999

\title{
INFINITE-DIMENSIONAL LIE ALGEBRAS OF GENERALIZED BLOCK TYPE
}

\author{
J. MARSHALL OSBORN AND KAIMING ZHAO
}

(Communicated by Lance W. Small)

\begin{abstract}
This paper investigates a class of infinite-dimensional Lie algebras over a field of characteristic 0 which are called here Lie algebras of generalized Block type, and which genereralize a class of Lie algebras originally defined by Richard Block. Under certain natural restrictions, this class of Lie algebras is shown to break into five subclasses. One of these subclasses contains all generalized Cartan type $K$ Lie algebras and some Lie algebras of generalized Cartan type $H$, and a second one is the class of Lie algebras of type $L$, which were previously defined and studied elsewhere by the authors. The other three types are hybrids of the first two types, and are new.
\end{abstract}

\section{INTRODUCTION}

Let $A$ be a torsion free abelian group, let $F$ be a field of characteristic 0 , and let $I=\{0,1, \ldots, n\}$. For each $i \in I$, let the maps $\phi_{i}: A \times A \rightarrow F$ be nonzero, and let $\delta_{i}$ for $i \in I$ be distinct elements of $A$. Set $\delta=\left(\delta_{0}, \ldots, \delta_{n}\right)$ and $\phi=\left(\phi_{0}, \ldots, \phi_{n}\right)$. Let $L=L(A, \delta, \phi)$ be the $F$-algebra with basis $\left\{e_{x} \mid x \in A\right\}$ and with the product

$$
\left[e_{x}, e_{y}\right]=\sum_{i=0}^{n} \phi_{i}(x, y) e_{x+y-\delta_{i}} .
$$

If $L$ is a Lie algebra, we call it a Lie algebra of generalized Block type. This is an obvious generalization of the class of algebras defined by Block in [B2], as well as the Lie algebras of type $L$ and generalized types $H, K$ studied in our papers [OZ1], [OZ2] and [OZ3].

A function $f(x, y)$ from $A^{2}$ to $F$ is called monomial in variables $x$ and $y$ if there exists a nonzero multi-additive function $g\left(x_{1}, \ldots, x_{n} ; y_{1}, \ldots, y_{m}\right)$ such that $f(x, y)$ is obtained from $g$ by setting $x_{1}=\cdots=x_{n}=x$ and $y_{1}=\cdots=y_{m}=y$. A function $f(x, y)$ from $A^{2}$ to $F$ is polynomial in variables $x$ and $y$ if it is a sum of monomial functions. We say that a function $f(x, y)$ from $A^{2}$ to $F$ is additive if $f(x, y)=g(x-y)$ for some additive map $g$.

Define $\operatorname{ker}\left(\phi_{i}\right)=\left\{x \in A \mid \phi_{i}(x, y)=0\right.$ for all $\left.y \in A\right\}$ for $i \in I$. We shall assume throughout in this paper that:

(A) the functions $\phi_{i}$ are polynomial;

(B) $\delta_{i}+\delta_{j}=\delta_{k}+\delta_{\ell} \Rightarrow\{i, j\}=\{k, \ell\}$ where $\{i, j\}$ denotes the set with $i$ and $j$ as its two elements; and

Received by the editors September 23, 1997.

1991 Mathematics Subject Classification. Primary 17B40, 17B65, 17B66, 17B68, 17B70.

(C)1999 American Mathematical Society 
(C) $\delta_{i} \notin \operatorname{ker}\left(\phi_{i}\right)$ for $i \in I$.

For any additive maps $\alpha, \beta: A \rightarrow F$ we shall denote the vector space spanned by $\alpha, \beta$ by $\langle\alpha, \beta\rangle$. We shall define the function $\alpha \wedge \beta: A^{2} \rightarrow F$ by $(\alpha \wedge \beta)(x, y)=$ $\alpha(x) \beta(y)-\alpha(y) \beta(x)$ for all $x, y \in A$. Our main result in this paper is the following:

Theorem 1.1. Suppose that $L(A, \delta, \phi)$ is an algebra defined in (1.1) satisfying Conditions (A), (B) and (C). Then $L$ is a Lie algebra, if and only if, there exist additive maps $\alpha_{i}, \beta_{i}, g_{i}: A \rightarrow F$ with $g_{i} \in\left\langle\alpha_{i}, \beta_{i}\right\rangle$ such that $\phi_{i}(x, y)=$ $\alpha_{i} \wedge \beta_{i}(x, y)+g_{i}(x-y)$ for all $i \in I$, and one of the following holds:

(I) all $\phi_{i}(x, y)=g_{i}(x-y)$ for $i \in I$;

(II) $I=I_{1} \cup I_{2}$, each $\phi_{i}$ for $i \in I_{1}$ is a multiple of an additive map $g: A \rightarrow F$ with $g\left(\delta_{i}-\delta_{j}\right)=0$ for all $i, j \in I$, and there exists an additive map $\beta: A \rightarrow F$ with $\beta\left(\delta_{i}-\delta_{j}\right)=0$ for all $i, j \in I$ such that $\alpha_{j} \wedge \beta_{j} \in F g \wedge \beta$ for each $j \in I_{2}$;

(III) $I=I_{1} \cup I_{2}$, each $\phi_{i}$ for $i \in I_{1}$ is a multiple of an additive map $g: A \rightarrow F$ with $g\left(\delta_{i}-\delta_{j}\right)=0$ for all $i, j \in I$, and there exists $b \in F$ and for each $j \in I_{2}$ there exist $a_{j} \in F$ and an additive map $\beta_{j}: A \rightarrow F$ such that $\phi_{j}(x, y)=g \wedge \beta_{j}(x, y)+$ $a_{j} \alpha(x-y)+b \beta_{j}(x-y)$

(IV) $I=I_{1} \cup I_{2}$, each $\phi_{i}(x, y)=g_{i}(x-y)$ with $g_{i}\left(\delta_{i}-\delta_{j}\right)=0$ for all $i \in I_{1}, j \in I_{2}$, each $\phi_{j}$ for $j \in I_{2}$ is not additive, there exist linearly independent additive maps $\alpha, \beta: A \rightarrow F$ such that $g_{j} \in\left\langle g_{i} \mid i \in I_{1}\right\rangle=\langle\alpha, \beta\rangle$ and $\alpha_{j} \wedge \beta_{j} \in F \alpha \wedge \beta$ for all $j \in I_{2}$;

$(V)$ each $\phi_{i}$ for $i \in I$ is not additive, and for any pair $i, j \in I$, one of the following holds:

(a) $\delta_{i} \in \operatorname{ker}\left(\phi_{j}\right)$ and $\delta_{j} \in \operatorname{ker}\left(\phi_{i}\right)$;

(b) $\operatorname{dim}\left(\left\langle\alpha_{i}, \beta_{i}\right\rangle \cap\left\langle\alpha_{j}, \beta_{j}\right\rangle\right)=1$ (we assume in this case that $\alpha_{i}=\alpha_{j}=\alpha$ ), $\alpha\left(\delta_{i}-\delta_{j}\right)=0, g_{i}=a_{i} \alpha+b \beta_{i}$ and $g_{j}=a_{j} \alpha+b \beta_{j}$ for some $a_{i}, a_{j}, b \in F$;

(c) there exist $a \in F^{*}$ and an additive map $\gamma: A \rightarrow F$ with $\gamma\left(\delta_{i}-\delta_{j}\right)=0$ such that $a \phi_{j}(x, y)=\phi_{i}(x, y)+\gamma(x-y)$.

\section{Some BASIC PROPERTIES OF POLYNOMIAL FUNCTIONS}

If a nonzero monomial $f(x, y): A^{2} \rightarrow F$ is obtained from a multi-additive function $g\left(x_{1}, \ldots, x_{n} ; y_{1}, \ldots, y_{m}\right)$ by setting $x_{1}=\cdots=x_{n}=x$ and $y_{1}=\cdots=$ $y_{m}=y$, then we say that $n$ is the degree of $f$ with respect to $x$ or the $x$-degree of $f, m$ is the degree of $f$ with respect to $y$ or the $y$-degree of $f$, and $m+n$ is the total degree of $f$. If $f(x, y): A^{2} \rightarrow F$ is a polynomial function, we shall call the highest degree of the summand monomials of $f$ with respect to $x$ the degree of $f$ with respect to $x$, the highest degree of the summand monomials of $f$ with respect to $y$ the degree of $f$ with respect to $y$, the highest total degree of the summand monomials of $f$ the total degree of $f$. Polynomial functions have a lot of properties which usual polynomials have.

Lemma 2.1. Suppose that $f(x, y), g(x, y): A^{2} \rightarrow F$ are polynomial functions and that $x_{0}, y_{0} \in A$. Then

(a) $f(x, y) g(x, y)=0 \Longrightarrow f(x, y)=0$ or $g(x, y)=0$;

(b) $f\left(x+x_{0}, y+y_{0}\right)$ is also a polynomial function, and $f\left(x+x_{0}, y+y_{0}\right)=$ $f(x, y)+f_{1}(x, y)$, where $f_{1}(x, y)$ has lower total degree than $f(x, y)$, has lower $x$ degree than $f(x, y)$, and has lower $y$-degree than $f(x, y)$. 
Proof. (a) By modifying the definition of monomial and polynomial in two variables, we can clearly get the definition of monomials and polynomials in one variable. The latter definition is needed in the first part of this proof which is

Claim 1. If $h_{1}(x), h_{2}(x): A \rightarrow F$ are nonzero polynomials in one variable, then $h_{1}(x) h_{2}(x) \neq 0$.

Fix $x_{1}, x_{2} \in A$ such that $h_{1}\left(x_{1}\right) h_{2}\left(x_{2}\right) \neq 0$. Suppose the degree of $h_{2}(x)$ is $k$. For any $l \in \mathbf{Z}$, we can write

$$
\begin{aligned}
& h_{1}\left(x_{1}+l x_{2}\right)=h_{1}\left(x_{1}\right)+l h_{1}^{\prime}(l), \\
& h_{2}\left(x_{1}+l x_{2}\right)=l^{k} h_{2}\left(x_{2}\right)+h_{2}^{\prime}(l),
\end{aligned}
$$

where $h_{1}^{\prime}(l)$ and $h_{2}^{\prime}(l)$ are polynomials in $l$ (also depending on $x_{1}, x_{2}$ ), and the degree of $h_{2}^{\prime}(l)$ with respect to $l$ is less than $k$ or $h_{2}^{\prime}(l)=0$. From $(2.2)$ we see that there exists an $N \in \mathbf{Z}$ such that $h_{2}\left(x_{1}+l x_{2}\right) \neq 0, \forall l>N$. If $h_{1}\left(x_{1}+l x_{2}\right)=0$ for all $l>N$, from (2.1) it follows that $h_{1}\left(x_{1}\right)=0$, which contradicts the choice of $x_{1}$. Thus there exists an $l_{0}>N$ such that $h_{1}\left(x_{1}+l_{0} x_{2}\right) \neq 0$. Therefore $h_{1}\left(x_{1}+l_{0} x_{2}\right) h_{2}\left(x_{1}+l_{0} x_{2}\right) \neq 0$. Consequently $h_{1}(x) h_{2}(x) \neq 0$. Our claim is proved.

Suppose that $f(x, y) \neq 0$ and $g(x, y) \neq 0$. Then there exist $x_{1}, x_{2}, y_{1}, y_{2} \in A$ such that $f\left(x_{1}, y_{1}\right) \neq 0$ and $g\left(x_{2}, y_{2}\right) \neq 0$. Then $f\left(x, y_{1}\right) \neq 0$ and $g\left(x, y_{2}\right) \neq 0$. It follows from Claim 1 that $f\left(x, y_{1}\right) g\left(x, y_{2}\right) \neq 0$. So there exists an $x_{0} \in A$ such that $f\left(x_{0}, y_{1}\right) g\left(x_{0}, y_{2}\right) \neq 0$. Thus $f\left(x_{0}, y\right) \neq 0$ and $g\left(x_{0}, y\right) \neq 0$. By Claim 1 again we see that $f\left(x_{0}, y\right) g\left(x_{0}, y\right) \neq 0$. It follows that there exists an $y_{0} \in A$ such that $f\left(x_{0}, y_{0}\right) g\left(x_{0}, y_{0}\right) \neq 0$. Therefore $f(x, y) g(x, y) \neq 0$. This completes the proof of (a).

(b) It is sufficient to show this for any monomial $f(x, y)$, and this follows easily from the definition.

The next result will be used frequently in the next section.

Lemma 2.2. Suppose that $\alpha_{1}, \alpha_{2}, \cdots, \alpha_{n}: A \rightarrow F$ are linearly independent additive maps.

(a) Let $f\left(X_{1}, \cdots, X_{n}\right) \in F\left[X_{1}, \cdots, X_{n}\right]$ be a polynomial in variables $X_{1}, \cdots, X_{n}$. If $f\left(\alpha_{1}(x), \alpha_{2}(x), \cdots, \alpha_{n}(x)\right)=0$ for all $x \in A$, then $f\left(X_{1}, \cdots, X_{n}\right)=0$;

(b) Let $f\left(X_{1}, \cdots, X_{2 n}\right) \in F\left[X_{1}, \cdots, X_{2 n}\right]$ be a polynomial in variables $X_{1}, \cdots$, $X_{2 n}$. If $f\left(\alpha_{1}(x), \alpha_{2}(x), \cdots, \alpha_{n}(x), \alpha_{1}(y), \alpha_{2}(y), \cdots, \alpha_{n}(y)\right)=0$ for all $x, y \in A$, then $f\left(X_{1}, \cdots, X_{2 n}\right)=0$.

Proof. (a) Suppose that $f\left(X_{1}, \cdots, X_{n}\right) \neq 0$. Since $\alpha_{1}, \alpha_{2}, \cdots, \alpha_{n}: A \rightarrow F$ are linearly independent, there exist $x_{1}, x_{2}, \cdots, x_{n} \in A$ such that the matrix

$$
M=\left(\begin{array}{cccc}
\alpha_{1}\left(x_{1}\right) & \alpha_{2}\left(x_{1}\right) & \cdots & \alpha_{n}\left(x_{1}\right) \\
\alpha_{1}\left(x_{2}\right) & \alpha_{2}\left(x_{2}\right) & \cdots & \alpha_{n}\left(x_{2}\right) \\
& \cdots & \cdots & \\
\alpha_{1}\left(x_{n}\right) & \alpha_{2}\left(x_{n}\right) & \cdots & \alpha_{n}\left(x_{n}\right)
\end{array}\right)
$$

is invertible. Let $x=l_{1} x_{1}+\cdots+l_{n} x_{n}$ where $l_{1}, l_{2}, \cdots, l_{n} \in \mathbf{Z}$. Then

$$
\left(\alpha_{1}(x), \alpha_{2}(x), \cdots, \alpha_{n}(x)\right)=\left(l_{1}, l_{2}, \cdots, l_{n}\right) M .
$$

Denote

$$
g\left(l_{1}, l_{2}, \cdots, l_{n}\right):=f\left(\alpha_{1}(x), \alpha_{2}(x), \cdots, \alpha_{n}(x)\right)=f\left(\left(l_{1}, l_{2}, \cdots, l_{n}\right) M\right) .
$$


Since $M$ is invertible and $f\left(X_{1}, \cdots, X_{n}\right) \neq 0$, it follows that $g\left(l_{1}, l_{2}, \cdots, l_{n}\right) \neq 0$ as a polynomial in $l_{1}, l_{2}, \cdots, l_{n}$. Thus there exist $k_{1}, k_{2}, \cdots, k_{n} \in \mathbf{Z}$ such that $g\left(k_{1}, k_{2}, \cdots, k_{n}\right) \neq 0$. If we set $x_{0}=k_{1} x_{1}+\cdots+k_{n} x_{n}$, we have $x_{0} \in A$ and $f\left(\alpha_{1}\left(x_{0}\right), \alpha_{2}\left(x_{0}\right), \cdots, \alpha_{n}\left(x_{0}\right)\right)=g\left(k_{1}, k_{2}, \cdots, k_{n}\right) \neq 0$. Therefore

$$
f\left(\alpha_{1}(x), \alpha_{2}(x), \cdots, \alpha_{n}(x)\right) \neq 0 .
$$

So (a) holds.

(b) Suppose that $f\left(X_{1}, \cdots, X_{2 n}\right) \neq 0$. By (a) we see that there exists $x_{0} \in A$ such that $f\left(\alpha_{1}\left(x_{0}\right), \alpha_{2}\left(x_{0}\right), \cdots, \alpha_{n}\left(x_{0}\right), X_{n+1}, \cdots, X_{2 n}\right) \neq 0$. Again by (a) we know that that there exists $y_{0} \in A$ such that

$$
f\left(\alpha_{1}\left(x_{0}\right), \alpha_{2}\left(x_{0}\right), \cdots, \alpha_{n}\left(x_{0}\right), \alpha_{1}\left(y_{0}\right), \alpha_{2}\left(y_{0}\right), \cdots, \alpha_{n}\left(y_{0}\right)\right) \neq 0 .
$$

Thus $f\left(\alpha_{1}(x), \alpha_{2}(x), \cdots, \alpha_{n}(x), \alpha_{1}(y), \alpha_{2}(y), \cdots, \alpha_{n}(y)\right) \neq 0$. So (b) is true.

\section{Lie algebras of generalized Block type}

Recall that we have assumed that the algebra $L=L(A, \delta, \phi)$ satisfies conditions (A), (B) and (C) stated in Section 1. In this section we shall determine the necessary and sufficient condition for the algebra $L$ to be a Lie algebra.

Lemma 3.1. If $L$ is a Lie algebra, then for each $i \in I$, we have

$$
\phi_{i}(x, y)=\phi_{i}^{\prime}(x, y)+g_{i}(x-y),
$$

where $g_{i}: A \rightarrow F$ is additive and $\phi_{i}^{\prime}: A \times A \rightarrow F$ is skew-symmetric and bi-additive.

Proof. We note that each $\phi_{i}$ must be anti-symmetric in order for the product to be anti-commutative. The remaining condition is the Jacobi identity for the product (1.1), which is equivalent to

$$
\begin{aligned}
0 & =\sum_{j=0}^{n} \sum_{i=0}^{n} \phi_{i}(x, y) \phi_{j}\left(x+y-\delta_{i}, z\right) e_{x+y+z-\delta_{i}-\delta_{j}} \\
& +\sum_{j=0}^{n} \sum_{i=0}^{n} \phi_{i}(y, z) \phi_{j}\left(y+z-\delta_{i}, x\right) e_{x+y+z-\delta_{i}-\delta_{j}} \\
& +\sum_{j=0}^{n} \sum_{i=0}^{n} \phi_{i}(z, x) \phi_{j}\left(z+x-\delta_{i}, y\right) e_{x+y+z-\delta_{i}-\delta_{j}} .
\end{aligned}
$$

Using Property (B), the Jacobi identity reduces to the following relation: for each pair $i, j \in I$,

$0=\phi_{i}(x, y) \phi_{j}\left(x+y-\delta_{i}, z\right)+\phi_{j}(x, y) \phi_{i}\left(x+y-\delta_{j}, z\right)+\phi_{i}(y, z) \phi_{j}\left(y+z-\delta_{i}, x\right)$

$$
\begin{array}{r}
+\phi_{j}(y, z) \phi_{i}\left(y+z-\delta_{j}, x\right)+\phi_{i}(z, x) \phi_{j}\left(z+x-\delta_{i}, y\right) \\
+\phi_{j}(z, x) \phi_{i}\left(z+x-\delta_{j}, y\right), \forall x, y, z \in A .
\end{array}
$$

We note first the special case of (3.2) where $j=i$ :

$$
\begin{array}{r}
\phi_{i}(x, y) \phi_{i}\left(x+y-\delta_{i}, z\right)+\phi_{i}(y, z) \phi_{i}\left(y+z-\delta_{i}, x\right) \\
+\phi_{i}(z, x) \phi_{i}\left(z+x-\delta_{i}, y\right)=0, \forall x, y, z \in A .
\end{array}
$$


Now we fix an $i \in I$, and let $\phi_{i}(x, y)=\sum_{j, k} f_{j, k}(x, y)$, where $f_{j, k}(x, y)$ is a function of degree $j$ in $x$ and degree $k$ in $y$. Let $n$ be the total degree of $\phi_{i}(x, y)$, and let $r$ be the $x$-degree of $\phi_{i}(x, y)$.

When we substitute for $\phi_{i}$ in (3.3), the terms for which the sum of the degrees in $x$ and in $y$ is maximal will have the sum of the degrees equal to $n+r$, since in each of the three terms in (3.3), one of the factors can have terms of combined degree $n$, and the other factor terms of combined degree only $r$. Further, the sum of the terms of combined degree $n+r$ must be zero. These terms by Lemma 2.1(b) give

$$
\begin{gathered}
0=\left(\sum_{j} f_{j, n-j}(x, y)\right)\left(\sum_{j} f_{r, j}(x+y, z)\right) \\
-\left(\sum_{j} f_{r, j}(y, z)\right)\left(\sum_{j} f_{j, n-j}(x, y)\right)-\left(\sum_{j} f_{r, j}(x, z)\right)\left(\sum_{j} f_{j, n-j}(x, y)\right) \\
=\left(\sum_{j} f_{j, n-j}(x, y)\right) \sum_{j}\left\{f_{r, j}(x+y, z)-f_{r, j}(y, z)-f_{r, j}(x, z)\right\} .
\end{gathered}
$$

Since $\sum_{j} f_{j, n-j}(x, y) \neq 0$, it follows from Lemma 2.1(a) that

$$
\sum_{j}\left[f_{r, j}(x+y, z)-f_{r, j}(y, z)-f_{r, j}(x, z)\right]=0
$$

for all $x, y, z \in A$. Thus $f_{r, j}(x+y, z)=f_{r, j}(x, z)+f_{r, j}(y, z)$ for all $j$ and all $x, y, z \in$ $A$, which says that the term of highest degree in $x$ in the expression for $\phi_{i}(x, y)$ is additive in the first variable. In other words, the maximal degree in the first variable of each $f_{j, k}(x, y)$ is 1 . By the antisymmetry of $\phi$, the same thing is true of the second variable. Thus, $\phi_{i}(x, y)=f_{1,1}(x, y)+f_{1,0}(x, y)+f_{0,1}(x, y)$. Since $f_{1,0}(x, y)$ is independent of the second variable $y$, and $f_{0,1}(x, y)$ is independent of the first variable $x$, we simply have $f_{1,0}(x, y)=f_{1,0}(x, 0)$ and $f_{0,1}(x, y)=f_{0,1}(0, y)$. Again by antisymmetry, $f_{0,1}(0, x)=-f_{1,0}(0, x)$, and so $\phi_{i}(x, y)=\phi_{i}^{\prime}(x, y)+g_{i}(x-y)$ where $\phi_{i}^{\prime}=f_{1,1}$ is skew-symmetric and bi-additive and $g_{i}(x)=f_{1,0}(x, 0)$ is additive.

From the proof of Lemma 3.1 we see that this lemma is true even without condition (C).

From now on we shall always assume that $\phi_{i}$ has the form in (3.1).

It follows from (3.1) that $\phi_{i}\left(x_{1}+x_{2}-x_{3}, y\right)=\phi_{i}\left(x_{1}, y\right)+\phi_{i}\left(x_{2}, y\right)+\phi_{i}\left(y, x_{3}\right)$. Using this in Equation (3.2), we get that

$$
\phi_{i}(x, y) \phi_{j}\left(z, \delta_{i}\right)+\phi_{i}(y, z) \phi_{j}\left(x, \delta_{i}\right)+\phi_{i}(z, x) \phi_{j}\left(y, \delta_{i}\right)+\phi_{j}(x, y) \phi_{i}\left(z, \delta_{j}\right)
$$

$$
+\phi_{j}(y, z) \phi_{i}\left(x, \delta_{j}\right)+\phi_{j}(z, x) \phi_{i}\left(y, \delta_{j}\right)=0, \forall x, y, z \in A .
$$

Note that $L(A, \delta, \phi)$ is a Lie algebra if and only if (3.4) holds for each pair $i, j \in I$.

In the next lemma, condition $(\mathrm{C})$ is not assumed.

Lemma 3.2. Suppose $L(A, \delta, \phi)$ is a Lie algebra.

(a) For a fixed $i \in I$, if $\delta_{i} \notin \operatorname{ker}\left(\phi_{i}\right)$, then there exist additive functions $\alpha_{i}, \beta_{i}, g_{i}$ : $A \rightarrow F$ with $g_{i} \in\left\langle\alpha_{i}, \beta_{i}\right\rangle$ such that $\phi_{i}(x, y)=\alpha_{i}(x) \beta_{i}(y)-\alpha_{i}(y) \beta_{i}(x)+g_{i}(x-y)$.

(b) For a fixed $i \in I$, if $\delta_{i} \in \operatorname{ker}\left(\phi_{i}\right)$, then $\phi_{i}(x, y)=\phi_{i}^{\prime}\left(x-\delta_{i}, y-\delta_{i}\right)$ for the bi-additive function $\phi_{i}^{\prime}$. 
Proof. (a) If $\phi_{i}$ is additive, we simply choose $\alpha_{i}=\beta_{i}:=g_{i}$. Then we get (a) in this case. Suppose then that $\phi_{i}$ is not additive. Setting $j=i$ in (3.4), we obtain

$$
\phi_{i}(x, y) \phi_{i}\left(z, \delta_{i}\right)+\phi_{i}(y, z) \phi_{i}\left(x, \delta_{i}\right)+\phi_{i}(z, x) \phi_{i}\left(y, \delta_{i}\right)=0 .
$$

Since $\delta_{i} \notin \operatorname{ker} \phi_{i}$, for any $z \in A$ with $\phi_{i}\left(z, \delta_{i}\right) \neq 0$, we have

$$
\phi_{i}(x, y)=c\left(\phi_{i}\left(x, \delta_{i}\right) \phi_{i}(y, z)-\phi_{i}\left(y, \delta_{i}\right) \phi_{i}(x, z)\right)
$$

where $c=-\phi_{i}\left(z, \delta_{i}\right)^{-1}$. If $\phi_{i}$ is bi-additive, i.e., $g_{i}=0$ in (3.1), we set $\alpha_{i}(x)=$ $c \phi_{i}\left(\delta_{i}, x\right)$ and $\beta(x)=\phi_{i}(z, x)$ in (3.5) to obtain $\phi_{i}(x, y)=\alpha_{i}(x) \beta_{i}(y)-\alpha_{i}(y) \beta_{i}(x)$, as desired.

If neither $\phi_{i}^{\prime}$ nor $g_{i}$ in (3.1) is identically 0 , let $\phi_{i}^{\prime}\left(x, \delta_{i}\right)=\gamma_{i}(x)-g_{i}(x)$ and $\phi_{i}^{\prime}(x, z)=\beta_{i}^{\prime}(x)-g_{i}(x)$. Then $(3.5)$ becomes

$$
\begin{gathered}
\phi_{i}(x, y)=c\left(\left(\gamma_{i}(x)-g_{i}\left(\delta_{i}\right)\right)\left(\beta_{i}^{\prime}(y)-g_{i}(z)\right)-\left(\gamma_{i}(y)-g_{i}\left(\delta_{i}\right)\right)\left(\beta_{i}^{\prime}(x)-g_{i}((z))\right)\right. \\
=c\left(\gamma_{i}(x) \beta_{i}^{\prime}(y)-\gamma_{i}(y) \beta_{i}^{\prime}(x)-\gamma_{i}(x-y) g_{i}(z)+\beta_{i}^{\prime}(x-y) g_{i}\left(\delta_{i}\right)\right) .
\end{gathered}
$$

By letting $\alpha_{i}(x)=g_{i}(x):=-c g_{i}(z) \gamma_{i}(x)+c g_{i}\left(\delta_{i}\right) \beta_{i}^{\prime}(x)$, and $\beta_{i}(x):=-g_{i}(z)^{-1} \beta_{i}^{\prime}(x)$, we see that (a) follows.

(b) From Lemma 3.1 we see that $\phi_{i}^{\prime} \neq 0$ in (3.1). Since $\delta_{i} \in \operatorname{ker}\left(\phi_{i}\right)$, from (3.1) we obtain that $\phi_{i}^{\prime}\left(x, \delta_{i}\right)+g_{i}(x)-g_{i}\left(\delta_{i}\right)=0$ for all $x \in A$. It follows that $g_{i}\left(\delta_{i}\right)=0$ and $g_{i}(x)=\phi_{i}^{\prime}\left(x, \delta_{i}\right)$. Thus (b) is true.

We recall that the function $\alpha \wedge \beta: A^{2} \rightarrow F$ for any additive maps $\alpha, \beta: A \rightarrow F$ is defined by $(\alpha \wedge \beta)(x, y)=\alpha(x) \beta(y)-\alpha(y) \beta(x)$. It is clear that, for any nonzero $\alpha^{\prime} \in\langle\alpha, \beta\rangle$, there exists $\beta^{\prime} \in\langle\alpha, \beta\rangle$ such that $\alpha \wedge \beta=\alpha^{\prime} \wedge \beta^{\prime}$.

Proposition 3.3. Suppose that $i, j \in I$ are fixed, that both $\phi_{i}(x, y)=\alpha_{i} \wedge \beta_{i}(x, y)+$ $g_{i}(x-y)$ and $\phi_{j}(x, y)=\alpha_{j} \wedge \beta_{j}(x, y)+g_{j}(x-y)$ are not additive, where $\alpha_{i}, \beta_{i} g_{i}, \alpha_{j}$, $\beta_{j}, g_{j}: A \rightarrow F$ are additive maps with $g_{i} \in\left\langle\alpha_{i}, \beta_{i}\right\rangle$ and $g_{j} \in\left\langle\alpha_{j}, \beta_{j}\right\rangle$. Then (3.4) holds, if and only if, one of the following holds:

(a) $\delta_{i} \in \operatorname{ker}\left(\phi_{j}\right)$ and $\delta_{j} \in \operatorname{ker}\left(\phi_{i}\right)$;

(b) $\operatorname{dim}\left(\left\langle\alpha_{i}, \beta_{i}\right\rangle \cap\left\langle\alpha_{j}, \beta_{j}\right\rangle\right)=1$, (we assume in this case that $\alpha_{i}=\alpha_{j}=\alpha$ ), and (a) is true or the following is true: $\alpha\left(\delta_{i}-\delta_{j}\right)=0, g_{i}=a_{i} \alpha+b \beta_{i}$ and $g_{j}=a_{j} \alpha+b \beta_{j}$ for some $a_{i}, a_{j}, b \in F$;

(c) there exist $a \in F^{*}$ and an additive map $\gamma: A \rightarrow F$ with $\gamma\left(\delta_{i}-\delta_{j}\right)=0$ such that $a \phi_{j}(x, y)=\phi_{i}(x, y)+\gamma(x-y)$.

Proof. $(\Rightarrow)$ Case 1. Suppose $\operatorname{dim}\left(\left\langle\alpha_{i}, \beta_{i}\right\rangle \cap\left\langle\alpha_{j}, \beta_{j}\right\rangle\right)=0$, i.e., $\alpha_{i}, \beta_{i}, \alpha_{j}, \beta_{j}$ are linearly independent.

By regarding $z$ as a fixed element in $A$ and $x, y$ as arbitrary elements, (3.4) has the form

$$
f\left(\alpha_{i}(x), \beta_{i}(x), \alpha_{j}(x), \beta_{j}(x), \alpha_{i}(y), \beta_{i}(y), \alpha_{j}(y), \beta_{j}(y)\right)=0, \forall x, y \in A
$$

for some polynomial

$$
f\left(X_{1}, X_{2}, X_{3}, X_{4}, Y_{1}, Y_{2}, Y_{3}, Y_{4}\right) \in F\left[X_{1}, X_{2}, X_{3}, X_{4}, Y_{1}, Y_{2}, Y_{3}, Y_{4}\right] .
$$

Then by Lemma $2.2, f\left(X_{1}, X_{2}, X_{3}, X_{4}, Y_{1}, Y_{2}, Y_{3}, Y_{4}\right)=0$. In particular, the coefficient of $X_{1} X_{2}$ in $f\left(X_{1}, X_{2}, X_{3}, X_{4}, Y_{1}, Y_{2}, Y_{3}, Y_{4}\right)$ is 0 , we obtain that $\phi_{j}\left(z, \delta_{i}\right)=0$ for any fixed $z \in A$. So $\delta_{i} \in \operatorname{ker}\left(\phi_{j}\right)$. By regarding $z$ as a fixed element in $A$ and $x, y$ as arbitrary elements, similar to the above argument by considering the coefficient of $\alpha_{j}(x) \beta_{j}(y)$ in (3.4), again from Lemma 2.2 we obtain that $\phi_{i}\left(z, \delta_{j}\right)=0$ for any fixed $z \in A$. So $\delta_{j} \in \operatorname{ker}\left(\phi_{i}\right)$. So (a) holds. 
Case 2. Suppose $\operatorname{dim}\left(\left\langle\alpha_{i}, \beta_{i}\right\rangle \cap\left\langle\alpha_{j}, \beta_{j}\right\rangle\right)=1$.

We may assume that $\alpha_{i}=\alpha_{j}=\alpha, g_{i}=a_{i} \alpha_{i}+b_{i} \beta_{i}, g_{j}=a_{j} \alpha_{i}+b_{j} \beta_{j}$ for some $a_{i}, b_{i}, a_{j}, b_{j} \in F$.

By comparing the total degree 3 parts on both sides of (3.4), we obtain

$$
\begin{aligned}
& \quad \phi_{i}^{\prime}(x, y)\left(\phi_{j}^{\prime}\left(z, \delta_{i}\right)+g_{j}(z)\right)+\phi_{i}^{\prime}(y, z)\left(\phi_{j}^{\prime}\left(x, \delta_{i}\right)+g_{j}(x)\right) \\
& \quad+\phi_{i}^{\prime}(z, x)\left(\phi_{j}^{\prime}\left(y, \delta_{i}\right)+g_{j}(y)\right)+\phi_{j}^{\prime}(x, y)\left(\phi_{i}^{\prime}\left(z, \delta_{j}\right)+g_{i}(z)\right) \\
& +\phi_{j}^{\prime}(y, z)\left(\phi_{i}^{\prime}\left(x, \delta_{j}\right)+g_{i}(x)\right)+\phi_{j}^{\prime}(z, x)\left(\phi_{i}^{\prime}\left(y, \delta_{j}\right)+g_{i}(y)\right)=0, \forall x, y, z \in A .
\end{aligned}
$$

By regarding $x, y$ as fixed elements in $A$ and $z$ as an arbitrary element, and by considering the coefficients of $\beta_{i}(z)$ in (3.7), from Lemma 2.2 we obtain that

$$
\alpha(y)\left(\phi_{j}^{\prime}\left(x, \delta_{i}\right)+g_{j}(x)\right)-\alpha(x)\left(\phi_{j}^{\prime}\left(y, \delta_{i}\right)+g_{j}(y)\right)+\phi_{j}^{\prime}(x, y)\left(-\alpha\left(\delta_{j}\right)+b_{i}\right)=0,
$$

i.e., $\left(\alpha(y) \beta_{j}(x)-\alpha(x) \beta_{j}(y)\right)\left(\alpha\left(\delta_{j}-\delta_{i}\right)+b_{j}-b_{i}\right)=0$. So

$$
\alpha\left(\delta_{j}-\delta_{i}\right)=b_{i}-b_{j} .
$$

It is not difficult to verify that (3.7) holds under the condition (3.8).

By comparing the total degree 2 parts on both sides of (3.4), we obtain

$$
\begin{array}{r}
-\phi_{i}^{\prime}(x, y) g_{j}\left(\delta_{i}\right)+g_{i}(x-y) \phi_{j}^{\prime}\left(z, \delta_{i}\right)-\phi_{i}^{\prime}(y, z) g_{j}\left(\delta_{i}\right) \\
+g_{i}(y-z) \phi_{j}^{\prime}\left(x, \delta_{i}\right)-\phi_{i}^{\prime}(z, x) g_{j}\left(\delta_{i}\right)+g_{i}(z-x) \phi_{j}^{\prime}\left(y, \delta_{i}\right) \\
-\phi_{j}^{\prime}(x, y) g_{i}\left(\delta_{j}\right)+g_{j}(x-y) \phi_{i}^{\prime}\left(z, \delta_{j}\right)-\phi_{j}^{\prime}(y, z) g_{i}\left(\delta_{j}\right) \\
+g_{j}(y-z) \phi_{i}^{\prime}\left(x, \delta_{j}\right)-\phi_{j}^{\prime}(z, x) g_{i}\left(\delta_{j}\right) \\
+g_{j}(z-x) \phi_{i}^{\prime}\left(y, \delta_{j}\right)=0, \forall x, y, z \in A .
\end{array}
$$

By setting $z=0$ in (3.9) we obtain

$$
\begin{gathered}
-\phi_{i}^{\prime}(x, y) g_{j}\left(\delta_{i}\right)+g_{i}(y) \phi_{j}^{\prime}\left(x, \delta_{i}\right)-g_{i}(x) \phi_{j}^{\prime}\left(y, \delta_{i}\right) \\
-\phi_{j}^{\prime}(x, y) g_{i}\left(\delta_{j}\right)+g_{j}(y) \phi_{i}^{\prime}\left(x, \delta_{j}\right)-g_{j}(x) \phi_{i}^{\prime}\left(y, \delta_{j}\right)=0,
\end{gathered}
$$

i.e.,

$$
\begin{gathered}
\left(\alpha(x) \beta_{i}(y)-\alpha(y) \beta_{i}(x)\right)\left(-\left(a_{j} \alpha\left(\delta_{i}\right)+b_{j} \beta_{j}\left(\delta_{i}\right)\right)+b_{i} \beta_{j}\left(\delta_{i}\right)+a_{j} \alpha\left(\delta_{j}\right)\right) \\
+\left(\alpha(x) \beta_{j}(y)-\alpha(y) \beta_{j}(x)\right)\left(-\left(a_{i} \alpha\left(\delta_{j}\right)+b_{i} \beta_{i}\left(\delta_{j}\right)\right)+b_{j} \beta_{i}\left(\delta_{j}\right)+a_{i} \alpha\left(\delta_{i}\right)\right) \\
+\left(\beta_{i}(x) \beta_{j}(y)-\beta_{i}(y) \beta_{j}(x)\right)\left(b_{i} \alpha\left(\delta_{i}\right)-b_{j} \alpha\left(\delta_{j}\right)\right)=0 .
\end{gathered}
$$

Since $\alpha, \beta_{i}, \beta_{j}$ are linearly independent, from Lemma 2.2 we conclude that

$$
\begin{gathered}
-\left(a_{j} \alpha\left(\delta_{i}\right)+b_{j} \beta_{j}\left(\delta_{i}\right)\right)+b_{i} \beta_{j}\left(\delta_{i}\right)+a_{j} \alpha\left(\delta_{j}\right)=0, \\
-\left(a_{i} \alpha\left(\delta_{j}\right)+b_{i} \beta_{i}\left(\delta_{j}\right)\right)+b_{j} \beta_{i}\left(\delta_{j}\right)+a_{i} \alpha\left(\delta_{i}\right)=0, \\
b_{i} \alpha\left(\delta_{i}\right)-b_{j} \alpha\left(\delta_{j}\right)=0 .
\end{gathered}
$$

Combining these equations with (3.8) we obtain

$$
\begin{gathered}
\alpha\left(\delta_{i}-\delta_{j}\right)=b_{j}-b_{i},\left(b_{j}-b_{i}\right)\left(a_{i}+\beta_{i}\left(\delta_{j}\right)\right)=0, \\
\left(b_{j}-b_{i}\right)\left(a_{j}+\beta_{j}\left(\delta_{i}\right)\right)=0, b_{i} \alpha\left(\delta_{i}\right)=b_{j} \alpha\left(\delta_{j}\right) .
\end{gathered}
$$


If $\alpha\left(\delta_{i}-\delta_{j}\right)=b_{j}-b_{i}=c \neq 0$, from the first and the fourth equations of (3.10), we see that $b_{i}=\alpha\left(\delta_{j}\right), b_{j}=\alpha\left(\delta_{i}\right)$. From the second and the third equations of (3.10) we get $a_{i}=-\beta_{i}\left(\delta_{j}\right), a_{j}=-\beta_{j}\left(\delta_{i}\right)$. Consequently $\phi_{i}(x, y)=\phi_{i}^{\prime}\left(x-\delta_{j}, y-\delta_{j}\right)$ and $\phi_{j}(x, y)=\phi_{i}^{\prime}\left(x-\delta_{i}, y-\delta_{i}\right)$. Then (a) is true. Otherwise we have $\alpha\left(\delta_{i}-\delta_{j}\right)=$ $b_{j}-b_{i}=0$. Thus (b) holds.

Case. Suppose $\operatorname{dim}\left(\left\langle\alpha_{i}, \beta_{i}\right\rangle \cap\left\langle\alpha_{j}, \beta_{j}\right\rangle\right)=2$.

We may assume that there exist an additive map $\gamma: A \rightarrow F$ and $a \in F^{*}$ such that $a \phi_{j}(x, y)=\phi_{i}(x, y)+\gamma(x-y)$. Then (3.4) becomes

$$
\begin{aligned}
&-\left(\phi_{i}(x, y)+\phi_{i}(y, z)+\phi_{i}(z, x)\right) \gamma\left(\delta_{i}\right) \\
&+\gamma(x-y) \phi_{i}\left(z, \delta_{j}\right)+\gamma(y-z) \phi_{i}\left(x, \delta_{j}\right)+\gamma(z-x) \phi_{i}\left(y, \delta_{j}\right)=0 .
\end{aligned}
$$

If $\gamma=0$, it is clear that (3.11) holds. So (c) is true in this case. Suppose then that $\gamma \neq 0$. We can choose an additive map $\gamma^{\prime}: A \rightarrow F$ such that $\phi_{i}^{\prime}=\gamma \wedge \gamma^{\prime}$. Then (3.11) becomes $\left(\phi_{i}^{\prime}(x, y)+\phi_{i}^{\prime}(y, z)+\phi_{i}^{\prime}(z, x)\right) \gamma\left(\delta_{i}-\delta_{j}\right)=0$. It follows that $\gamma\left(\delta_{i}-\delta_{j}\right)=0$. Thus (c) holds.

$(\Leftarrow)$ If condition (a), (b) or (c) holds, it is not difficult to verify that (3.4) holds, i.e., $L(A, \delta, \phi)$ is a Lie algebras. We omit the details here.

Proposition 3.4. Suppose that $i, j \in I$ are fixed, that $\phi_{i}(x, y)=g_{i}(x-y)$ and $\phi_{j}(x, y)=\alpha_{j} \wedge \beta_{j}(x, y)+g_{j}(x-y)$, where $g_{i}, \alpha_{j}, \beta_{j}, g_{j}: A \rightarrow F$ are additive maps with $g_{j} \in\left\langle\alpha_{j}, \beta_{j}\right\rangle$ and $\alpha_{j} \wedge \beta_{j} \neq 0$. Then (3.4) holds, if and only if, $g_{i} \in\left\langle\alpha_{j}, \beta_{j}\right\rangle$ and $g_{i}\left(\delta_{i}\right)=g_{i}\left(\delta_{j}\right)$.

Proof. $(\Rightarrow)$ From (3.4) we obtain that

$$
\begin{aligned}
& g_{i}(x-y) \phi_{j}^{\prime}\left(z, \delta_{i}\right)+g_{i}(y-z) \phi_{j}^{\prime}\left(x, \delta_{i}\right)+g_{i}(z-x) \phi_{j}^{\prime}\left(y, \delta_{i}\right) \\
+ & \phi_{j}^{\prime}(x, y) g_{i}\left(z-\delta_{j}\right)+\phi_{j}^{\prime}(y, z) g_{i}\left(x-\delta_{j}\right)+\phi_{j}^{\prime}(z, x) g_{i}\left(y-\delta_{j}\right)=0 .
\end{aligned}
$$

Comparing the total degree 3 parts on both sides of (3.12) yields

$$
\phi_{j}^{\prime}(x, y) g_{i}(z)+\phi_{j}^{\prime}(y, z) g_{i}(x)+\phi_{j}^{\prime}(z, x) g_{i}(y)=0 .
$$

By fixing $x, y \in A$ with $\phi_{j}^{\prime}(x, y) \neq 0$, we obtain that

$$
g_{i}(z)=-\phi_{j}^{\prime}(x, y)^{-1}\left(\phi_{j}^{\prime}(y, z) g_{i}(x)+\phi_{j}^{\prime}(z, x) g_{i}(y)\right), \forall z \in A .
$$

Thus we deduce that $g_{i} \in\left\langle\alpha_{j}, \beta_{j}\right\rangle$. Without loss of generality we may assume that $g_{i}=\alpha_{j}$.

By comparing the total degree 2 parts on both sides of (3.12), we obtain

$$
\begin{gathered}
g_{i}(x-y) \phi_{j}^{\prime}\left(z, \delta_{i}\right)+g_{i}(y-z) \phi_{j}^{\prime}\left(x, \delta_{i}\right)+g_{i}(z-x) \phi_{j}^{\prime}\left(y, \delta_{i}\right) \\
-\left(\phi_{j}^{\prime}(x, y)+\phi_{j}^{\prime}(y, z)+\phi_{j}^{\prime}(z, x)\right) g_{i}\left(\delta_{j}\right)=0 .
\end{gathered}
$$

Using $\alpha_{j}=g_{i}$, we get $\left(g_{i}(x-y) \beta_{j}^{\prime}(z)+g_{i}(y-z) \beta_{j}^{\prime}(x)+g_{i}(z-x) \beta_{j}^{\prime}(y)\right) g_{i}\left(\delta_{i}-\delta_{j}\right)=0$. Setting $z=0$ in the above equation yields $\left(g_{i}(y) \beta_{j}(x)-g_{i}(x) \beta_{j}(y)\right) g_{i}\left(\delta_{i}-\delta_{j}\right)=0$. Since $g_{i}, \beta_{j}$ are linearly independent, from Lemma 2.2 we deduce that $g_{i}\left(\delta_{i}-\delta_{j}\right)=0$.

$(\Leftarrow)$ This follows by direct verification. We leave the details to the reader.

Now we are ready to prove our main result, Theorem 1.1.

Proof of Theorem 1.1. $(\Rightarrow)$ From Lemma 3.2(a) we see that there exist additive maps $\alpha_{i}, \beta_{i}, g_{i}: A \rightarrow F$ with $g_{i} \in\left\langle\alpha_{i}, \beta_{i}\right\rangle$ such that $\phi_{i}(x, y)=\alpha_{i} \wedge \beta_{i}(x, y)+g_{i}(x-y)$ for all $i \in I$. Denote $\phi_{i}^{\prime}=\alpha_{i} \wedge \beta_{i}$ for all $i \in I$. Then $\phi_{i}(x, y)=\phi_{i}^{\prime}(x, y)+g_{i}(x-y)$. 
Case 1. Suppose that all of $\phi_{i}$ are additive. This is (I).

Case 2. Suppose that $I=I_{1} \cup I_{2}$, each $\phi_{i}$ for $i \in I_{1}$ is a multiple of an additive map $g: A \rightarrow F$, and each $\phi_{j}$ for $j \in I_{2}$ is not additive.

For any $i \in I_{1}$ and any $j \in I_{2}$, from Proposition 3.4 we may assume that $\alpha_{j}=g$ and $g\left(\delta_{i}\right)=g\left(\delta_{j}\right)$. Then $g\left(\delta_{i}\right)=g\left(\delta_{j}\right)$ for all $i . j \in I$.

For any $i \in I_{2}$ we know that $\phi_{i}=g \wedge \beta_{i}+g_{i}(x-y)$ and $g\left(\delta_{i}\right)=g\left(\delta_{j}\right)$ for any $j \in I$. If there exist $i_{0}, j_{0} \in I_{2}$ such that $g, \beta_{i_{0}}, \beta_{j_{0}}$ are linearly independent, from Proposition 3.3(b) we deduce that there exist $a_{i_{0}}, a_{j_{0}}, b \in F$ such that $g_{i_{0}}=$ $a_{i_{0}} g+b \beta_{i_{0}}$ and $g_{j_{0}}=a_{j_{0}} g+b \beta_{j_{0}}$. For any $k \in I_{2} \backslash\left\{i_{0}, j_{0}\right\}$ we know that $g, \beta_{i_{0}}, \beta_{k}$ are linearly independent, or that $g, \beta_{k}, \beta_{j_{0}}$ are linearly independent. We may assume that $g, \beta_{i_{0}}, \beta_{k}$ are linearly independent. Since $g\left(\delta_{i_{0}}\right)=g\left(\delta_{k}\right)$, again from Proposition $3.3(\mathrm{~b})$ we see that there exist $a_{k} \in F$ such that $g_{k}=a_{k} g+b \beta_{k}$. Thus (III) holds. Now we suppose that $g, \beta_{i}, \beta_{j}$ are linearly dependent for all $i, j \in I_{2}$. If there exist $i_{0}, j_{0} \in I_{2}$ such that $\beta\left(\delta_{i_{0}}-\delta_{j_{0}}\right) \neq 0$. From Proposition 3.3(b) we deduce that there exist $a_{i_{0}}, a_{j_{0}}, b \in F$ such that $g_{i_{0}}=a_{i_{0}} g+b \beta_{i_{0}}$ and $g_{j_{0}}=a_{j_{0}} g+b \beta_{j_{0}}$. For any $k \in I_{2} \backslash\left\{i_{0}, j_{0}\right\}$ we know that $\beta\left(\delta_{k}-\delta_{j_{0}}\right) \neq 0$ or $\beta\left(\delta_{i_{0}}-\delta_{k}\right) \neq 0$. We may assume that $\beta\left(\delta_{k}-\delta_{j_{0}}\right) \neq 0$. Again from Proposition 3.3 (c) we know that there exist $a_{k} \in F$ such that $g_{k}=a_{k} g+b \beta_{k}$. Thus (III) holds. If $\beta\left(\delta_{i}-\delta_{j}\right) \neq 0$ for all $i, j \in I_{2}$, from Proposition 3.3(c), then (II) holds.

Case 3. Suppose that $I=I_{1} \cup I_{2}$, each $\phi_{i}(x, y)=g_{i}(x-y)$ for $i \in I_{1}$ is additive, each $\phi_{j}$ for $j \in I_{2}$ is not additive, and there exist linearly independent additive maps $\alpha, \beta: A \rightarrow F$ such that $\left\langle g_{i} \mid i \in I_{1}\right\rangle=\langle\alpha, \beta\rangle$.

For any $i \in I_{1}$ and any $j \in I_{2}$, from Proposition 3.4 we see that $g_{i} \in\left\langle\alpha_{j}, \beta_{j}\right\rangle$ and $g_{i}\left(\delta_{i}\right)=g_{i}\left(\delta_{j}\right)$. Since $\left\langle g_{i} \mid i \in I_{1}\right\rangle=\langle\alpha, \beta\rangle$. Then $\left\langle\alpha_{j}, \beta_{j}\right\rangle=\langle\alpha, \beta\rangle$ for any $j \in I_{2}$. Also we have $\alpha\left(\delta_{i}\right)=\alpha\left(\delta_{j}\right)$ and $\beta\left(\delta_{i}\right)=\beta\left(\delta_{j}\right)$ for all $i, j \in I_{2}$.

For any $i \in I_{2}$ we know that there exists $c_{i} \in F^{*}$ such that

$$
\phi_{i}=c_{i} \alpha \wedge \beta+g_{i}(x-y)
$$

and $\alpha\left(\delta_{i}\right)=\alpha\left(\delta_{j}\right)$ and $\beta\left(\delta_{i}\right)=\beta\left(\delta_{j}\right)$ for any $j \in I_{2}$. From Propositon 3.3(c) we see that (3.4) holds for all arbitrary $g_{i}, g_{j} \in\langle\alpha, \beta\rangle$ and $i, j \in I_{2}$. This shows that (IV) holds.

Case 4. Suppose that each $\phi_{i}$ for $i \in I$ is not additive.

From Proposition 3.4 we see that $(\mathrm{V})$ holds.

$(\Leftarrow)$ If there exist additive maps $\alpha_{i}, \beta_{i}, g_{i}: A \rightarrow F$ with $g_{i} \in\left\langle\alpha_{i}, \beta_{i}\right\rangle$ such that

$$
\phi_{i}(x, y)=\alpha_{i} \wedge \beta_{i}(x, y)+g_{i}(x-y)
$$

for all $i \in I$, and one of $(\mathrm{I})-(\mathrm{V})$ holds, we see that $\phi_{i}(x, y)$ is skew-symmetric. It suffices to verify that (3.4) holds for all $i, j \in I$. But this is straightforward. We omit the details.

For the "only if" part, we still can get Lie algebras even without condition (B) or condition $(\mathrm{C})$.

Algebras in (I) are called Type L Lie algebras. For more details about Type L Lie algebras, we refer the reader to our paper [OZ3]. All generalized Cartan type $\mathrm{K}$ Lie algebras and some generalized Cartan type $\mathrm{H}$ Lie algebras are contained in $(\mathrm{V})$. 


\section{REFERENCES}

[B1] R. Block, New simple Lie algebras of prime characteristic, Trans. Amer. Math. Soc., 72(1958), 421-449. MR 20:6446

[B2] R. Block, On torsion-free abelian groups and Lie algebras, Proc. Amer. Math. Soc., 9(1958), 613-620. MR 20:3913

[DZ1] D.Z. Djokovic and K. Zhao, Derivations, isomorphisms, and second cohomology of generalized Block algebras, Algebra Colloquium, Vol.3, 3(1996).

[DZ2] D.Z. Djokovic and K. Zhao, Some infinite dimensional simple Lie algebras related to those of Block, J. Pure and Applied Algebra, in press.

[OZ1] J.M. Osborn and K. Zhao, Generalized Poisson brackets and Lie algebras of type H in characteristic 0, Math. Z., 229(1998), No. 4.

[OZ2] J.M. Osborn and K. Zhao, Generalized Cartan type K Lie algebras in characteristic 0, Comm. Alg., 25(1997), 3325-3360. CMP 97:17

[OZ3] J.M. Osborn and K. Zhao, Infinite dimensional Lie algebras of type L, preprint, 1997.

Department of Mathematics, University of Wisconsin, Madison, Wisconsin 53706

E-mail address: osborn@math.wisc.edu

Institute of Systems Science, Academia Sinica, Beijing, 100080, China

E-mail address: zhao@iss06.iss.ac.cn (or zhao@math.wisc.edu) 